\title{
Association Between Driving in the Summer and COVID-19 Mortality in the Autumn
}

J Gen Intern Med 36(11):3632-5

DOI: $10.1007 / \mathrm{s} 11606-021-07074-\mathrm{Z}$

(C) Society of General Internal Medicine 2021

\section{INTRODUCTION}

Behavioral interventions can be successful methods to control a pandemic. ${ }^{1}$ In response to COVID-19, onethird of the world's nations instituted formal lockdowns. ${ }^{1}$ We previously showed that nations that reduced mobility experienced less COVID-19 mortality during the early months of the pandemic. ${ }^{2}$ Severe restrictions are not sustainable; however, premature loosening of restrictions can lead to a COVID-19 resurgence. ${ }^{1}$ In particular, many nations eased mobility restrictions in the summer of 2020 . Herein, we reassessed the association between mobility and COVID-19 mortality by examining changes of driving distances in the summer and COVID-19 mortality in the autumn.

\section{METHODS}

We examined the same original 36 nations from our previous study of the first wave of the COVID-19 pandemic. $^{2} \mathrm{We}$ calculated the mean change in mobility from baseline (driving distances on January 13, 2020) for each nation during the summer (June through August 2020) using Apple Mobility Trends. ${ }^{3}$ For each nation, we collected data on COVID-19 mortality during the autumn (September through November 2020) using Our World in Data. ${ }^{4}$ Excess mortality was estimated by dividing the total number of COVID-19 deaths by

Received December 11, 2020

Accepted July 21, 2021

Published online August 6, 2021 the total population size of each nation. In a secondary analysis, we assessed the association between changes in driving distance and changes in COVID-19 cases. As in earlier studies $^{2}$, we used the Spearman rank correlation coefficient to test the association between the reduction in driving and subsequent changes in COVID-19 mortality rate in each nation.

\section{RESULTS}

The majority of nations $(n=26,72 \%)$ demonstrated increased driving distances in the summer compared to their own earlier baseline. In the autumn, we also found significant variation in COVID-19 mortality rates. For example, the Czech Republic had the highest mortality $(73.5$ per 100,000) and Singapore had the lowest mortality $(0.03$ per 100,000$)$. As hypothesized (Fig. 1), we observed a significant association between increased driving distances and higher COVID-19 mortality $(r=0.35, p=0.036)$. In addition, we observed a significant association between increased driving and higher COVID-19 cases $(r=0.48, p=0.003$ ) (Fig. 2). These trends suggested that, for the USA, a $10 \%$ increase in driving distances might have contributed to an additional 4,100 deaths and 459,000 cases.

Sensitivity analyses using shorter and longer time periods for assessing changes in mean driving distances found similar correlations of increased driving with increased COVID-19 mortality. In an additional sensitivity analysis restricted to 19 of the nations deemed by the WHO in the top public health systems, we observed consistent findings $(r=0.37) .{ }^{5}$ In a further analysis restricted to the 21 nations with data on public transit, we found no significant correlation between increased use of public transit and increased COVID-19 mortality $(r=0.07)$. 


\section{DISCUSSION}

We found that nations exhibiting increased driving in the summer experienced more COVID-19 mortality in the autumn. These findings support previous work suggesting that communities with greater adherence to mobility restrictions experienced lower COVID-19 mortality. ${ }^{2,6}$ However, our findings are unlikely causal because population mobility may be one of several factors associated with COVID-19 mortality.

The stronger association with COVID-19 incidence rather than COVID-19 mortality suggests community spread of infection may be related to human mobility. This differential also highlights the potential greater importance of demographics, healthcare capacity, and other factors for influencing

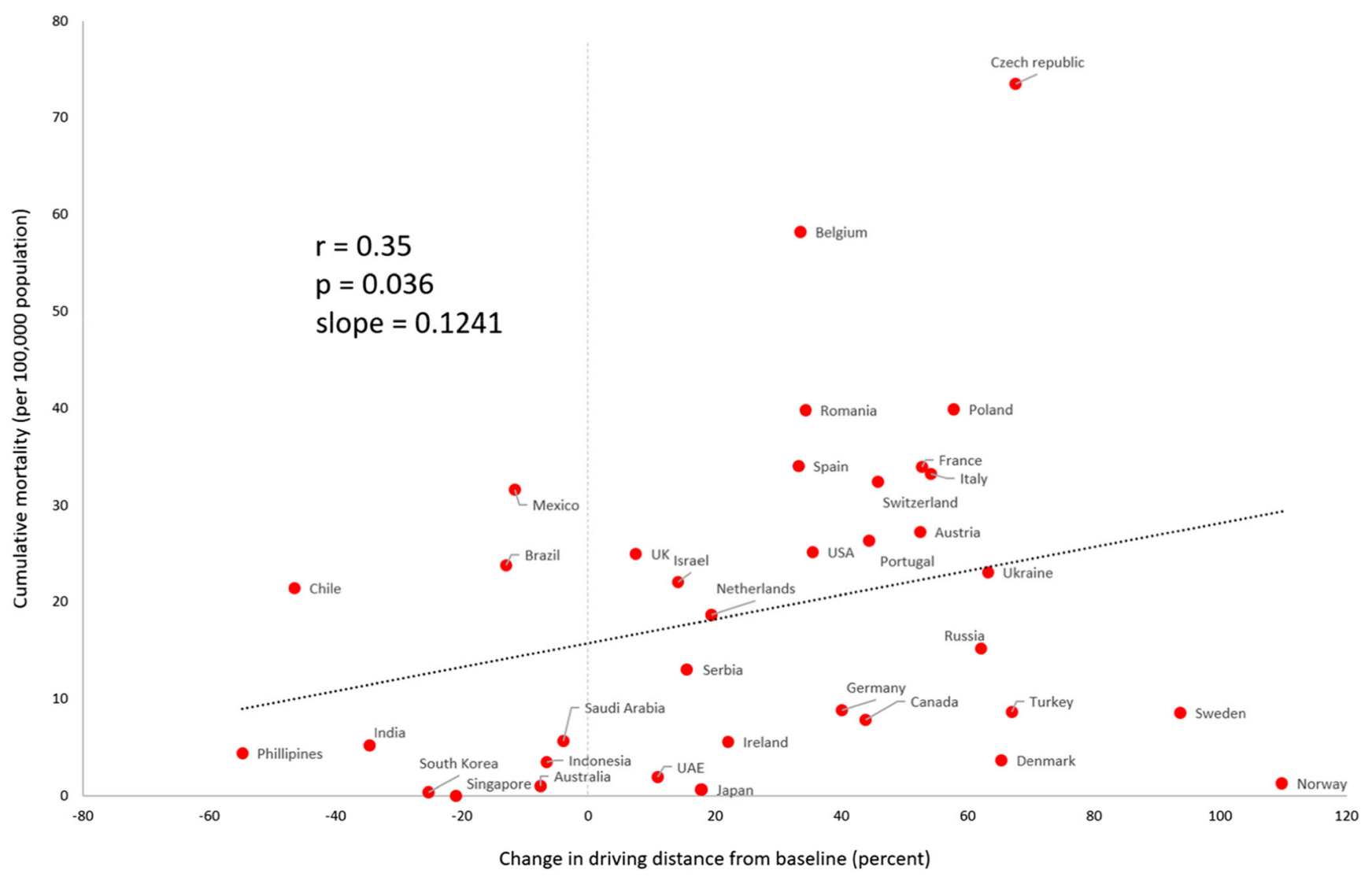

Figure 1 Association between driving distances and COVID-19 mortality. Nations to the right of zero show increased driving and nations to the left show decreased driving. $Y$-axis denotes total additional deaths from COVID in autumn for each nation adjusted for total population size. Correlation coefficient ( $r$ ) based on the Spearman rank statistic. Overall data show a significant association between increased driving and increased mortality. 


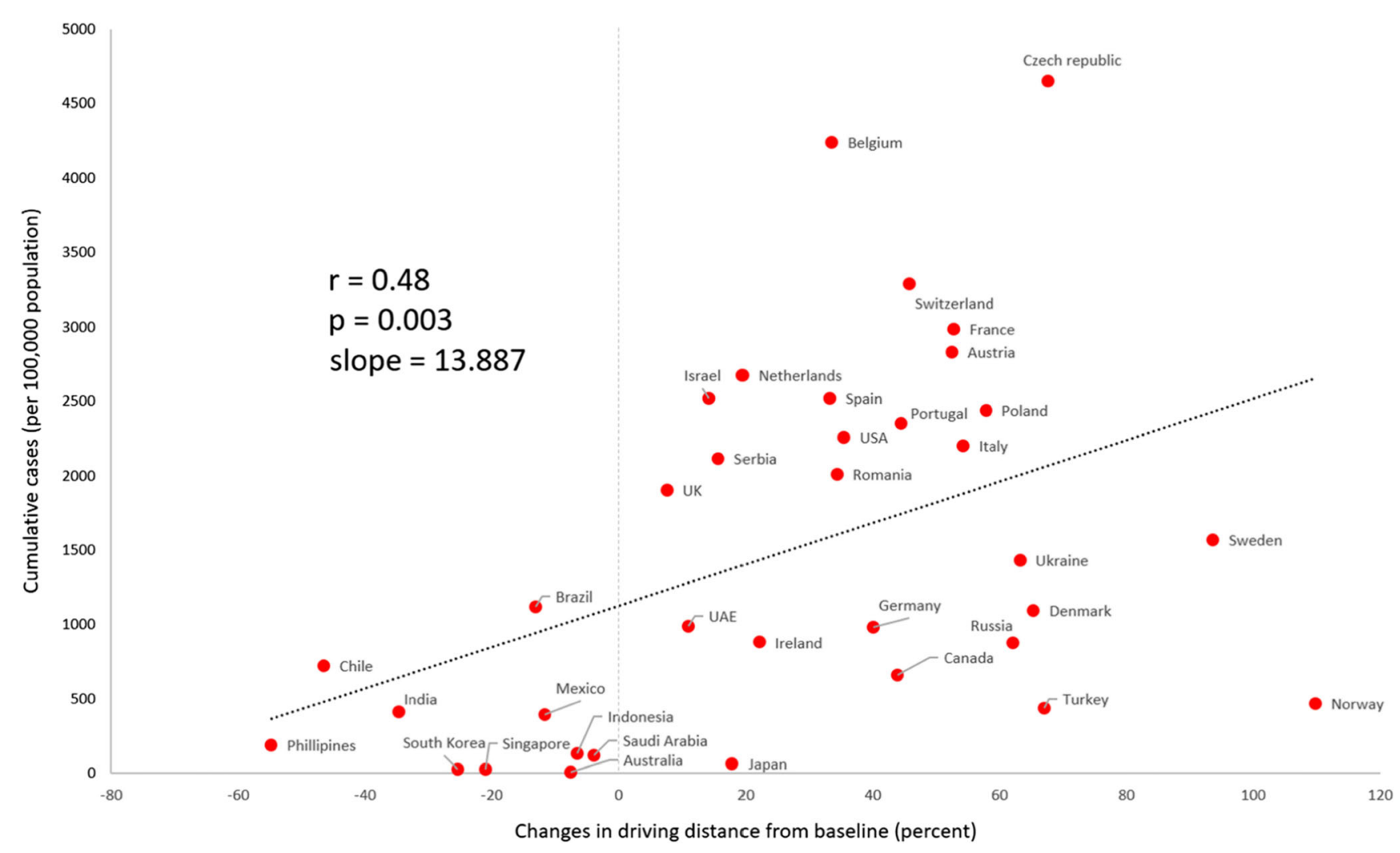

Figure 2 Association between driving distances and COVID-19 cases. Countries to the right of zero show increased driving and nations to the left show decreased driving. $Y$-axis denotes total additional cases from COVID in autumn for each nation adjusted for total population size. Correlation coefficient $(r)$ based on the Spearman rank statistic. Overall data show a significant association between increased driving and increased COVID-19 case counts.

COVID-19 case fatality. As well, more research on countries such as Norway is needed to better understand how a few outlier nations sustained low COVID-19 mortality despite having substantial increases in driving distances in the summer.

One limitation of our study is the uncertainty around whether some nations loosened restrictions in response to excellent initial control of the virus. Such reverse causality is possible, since our analysis assessed outcomes three months after assessment of driving patterns without adjustment for other potential confounders. As well, we were unable to account for whether people were traveling by car instead of plane because we lacked data on alternate modes of transport. Additional limitations include the inability to determine vehicle occupancy, the purpose of individual trips, or whether the driving was for private or commercial purposes.

In conclusion, our data further support the potential for controlling community spread of a pandemic through 
mobility restrictions in addition to other behavioral interventions.

Jonathan S. Zipursky, MD FRCPC ${ }^{1}$

Donald A. Redelmeier, MD FRCPC MSHSR FACP ${ }^{1,2,3,4,5}$

${ }^{1}$ Department of Medicine, University of Toronto,

Toronto, Canada

${ }^{2}$ Evaluative Clinical Sciences Program, Sunnybrook Research Institute,

Toronto, Canada

${ }^{3}$ Institute for Clinical Evaluative Sciences,

Toronto, Canada

${ }^{4}$ Division of General Internal Medicine, Sunnybrook Health Sciences Centre,

Toronto, Canada

${ }^{5}$ Center for Leading Injury Prevention Practice Education \& Research,

Toronto, Canada

Corresponding Author: Donald A. Redelmeier, MD FRCPC MSHSR FACP; Division of General Internal Medicine, Sunnybrook Health Sciences Centre, Toronto, Canada (e-mail: dar@ices.on.ca).

\section{Declarations:}

Conflict of Interest: JSZ reports payments for medicolegal opinions regarding the safety and effectiveness of drugs outside the submitted work. DAR reports no conflicts of interest.

\section{REFERENCES}

1. Han E, Tan MMJ, Turk E, et al. Lessons learnt from easing COVID-19 restrictions: an analysis of countries and regions in Asia Pacific and Europe. Lancet (London, England). 2020;396(10261):1525-1534. doi:https://doi.org/10.1016/S0140-6736(20)32007-9

2. Zipursky JS, Redelmeier DA. Mobility and Mortality During the COVID-19 Pandemic. J Gen Intern Med. 2020;35:3100-1. https://doi.org/10.1007/ s11606-020-05943-7

3. Apple Maps. Mobility Trends Reports. https://www.apple.com/covid19/ mobility. Published 2020. Accessed 1 Dec 2020

4. Coronavirus Pandemic (COVID-19) - the data. Our World in Data. https:// ourworldindata.org/coronavirus-data. Published 2020. Accessed December 1, 2020.

5. Tandon A, Murray CJL, Lauer JA, Evans DB. Measuring Overall Health System Performance for 191 Countries. GPE Discussion Paper Series: No. 30. EIP/GPE/EQC. World Health Organization

6. Jüni P, Rothenbühler M, Bobos P, et al. Impact of climate and public health interventions on the COVID-19 pandemic: a prospective cohort study. Can Med Assoc J. 2020;192(21):E566-E573. doi:https://doi.org/10.1503/ cmaj. 200920

Publisher's Note: Springer Nature remains neutral with regard to jurisdictional claims in published maps and institutional affiliations. 